\title{
Physiological and Biochemical Effect of Endurance Training of Altitude Training on Fixed Maximal Oxygen Expenditure in Middle and Long Distance Runners
}

\author{
Lang QIN and Jin YIN \\ School of Sports Medicine and Health, Chengdu Sport University, Chengdu, Sichuan, \\ China \\ ${ }^{*}$ Corresponding author
}

Keywords: Altitude training, Physiological and biochemical effect, Exhausted run, live moderate and training high.

\begin{abstract}
The purpose of this study was to investigate the exercise intensity of endurance training of altitude training under the plain and plateau by means of the fixed maximal oxygen expenditure. Eight middle and long distance runners voluntarily participated in this study, the ergmeter bike and telemetry cardiopulmonary function system were used in the endurance test in Chengdu and Kangding, and the oxygen uptake, heart rate, power output, oxhemoglobin saturation by pulse oximetry $\left(\mathrm{SPO}_{2}\right)$, and blood lactic acid were tested. The results indicated that the altitude training can reduce the power output and $\mathrm{SPO}_{2}$, the power output and heart rate were significant affected under long term altitude training, and the heart rate and blood lactic acid seem have not been affected by training environmental factors. The findings of this study revealed that taking the heart rate as monitor criteria in moderate altitude training was an appropriate way, and the power output criteria may under- or over-estimate the exercise intensity in altitude training.
\end{abstract}

\section{Introduction}

Altitude training have been widely used in sports training, in the recent literature, the main method are live low and train high, and live high and train low. The coach can make their train program according to the requirement of training protocol.

In the recent literature, the authors reviewed some former findings of some researchers. Diebel et al. estimated the impact of altitude training on BL changes immediately following an incremental treadmill test and during recovery before and after 10-day altitude training at approximately 1828 meters. Their findings indicated that a 10-day altitude intervention at 1828 meters may benefit varsity cross-country runners. The higher post-exercise BL may be attributed to more anaerobic contributions Lower HR may suggest a larger stroke volume and/or more efficient $\mathrm{O}_{2}$ carrying capacity [1]. Woods et al. determined whether 4 weeks of classical altitude training affects RMR in middle-distance runners. Ten highly trained athletes were recruited for 4 weeks of endurance training undertaking identical programs at either $2200 \mathrm{~m}$ in Flagstaff, Arizona or $600 \mathrm{~m}$ in Canberra, Australia . The findings determined that the altitude exposure may increase RMR and enhance training adaptation.[2] Stickford et al. determined if select kinematic variables changed in a group of elite distance runners after 4 wks of altitude training. The study indicated that the running mechanics are not affected by chronic altitude training in elite distance runners. The data suggest that either chronic training at altitude truly has no effect on running mechanics or completing the live high-train low model of altitude training, 
where higher-velocity workouts are completed at lower elevations, mitigates any negative mechanical adaptations that may be associated with chronic training at slower speeds. [3] Warren et al. investigated the effects of an elevation training mask (ETM) on the VO2max of male Reserve Officers Training Corps cadets. This study concluded that the ETM did not cause a significant increase in $\mathrm{VO}_{2 \max }$ under the training conditions of this study. [4] Bor-Kucukatay et al. investigated the possible alterations in hemorheological parameters in response to altitude training. The results of this study show that, living (LHC) and training at altitude (LHTH) seems more advantageous in hemorheological point of view. [5] Carr et al. examined effects of low altitude training and a live-high: train-low protocol (combining both natural and simulated modalities) on haemoglobin mass, maximum oxygen consumption (VO2max), time to exhaustion, and submaximal exercise measures. We recommend low altitude $(1380 \mathrm{~m})$ combined with sleeping in altitude tents as one effective alternative to traditional altitude training methods, which can improve Hbmass.[6] Siewierski et al. estimated changes in blood morphology caused by participation of record-seeking swimmers in a high altitude training camp at 2,300 $\mathrm{m}$ above sea level and to assess their performance during major competitions before and after the camp. The response of the examined swimmers from the Polish Olympic Team to the high altitude training (at 2,300 $\mathrm{m}$ above sea level) was represented by an almost three-fold increase in blood reticulocyte count during the first micro-cycle of training as well as by an elevated erythrocyte count, and haemoglobin and haematocrit levels estimated after completion of the training, as compared to the results obtained before the camp. Six out of eight subjects improved their performance in major competitions organized after the training camp, and four of them broke their personal bests. [7] Suchy et al tested the influence of a ten day altitude training camp on performance in well-trained adolescent cross-country skiers. In the model we were testing, the ten day altitude camp was to verify a positive effect on adolescents in acute improvement of their performance ten days after their return to low altitude. [8] McLean et al. examined changes in 2000-m time-trial running performance (TT), hemoglobin mass (Hbmass), and intramuscular carnosine content of elite Australian Football players after a preseason altitude camp. A preseason altitude camp improved TT performance and Hbmass in elite AF players to a magnitude similar to that demonstrated by elite endurance athletes undertaking altitude training. The individual responsiveness of both TT and Hbmasswas approximately half the groups mean effect, indicating that most players gained benefit. The maintenance of running performance for $4 \mathrm{wks}$, despite Hbmass returning to baseline, suggests that altitude training is a valuable preparation for AF players leading into the competitive season. [9] Garvican et al determined the time course of hemoglobin mass (Hbmass) to natural altitude training, Their results suggest that in elite cyclists, Hbmass increases progressively with 3 weeks of natural altitude exposure, with greater increases expected as exposure persists. [10] Robach, P. \& Lundby, C. examined the relevance of live high-train low altitude training for elite athletes with already high total hemoglobin mass. They mentioned that LHTL was adopted in training programs of sport federations and is accepted as the most effectivealtitude training regime. They assert that LHTL may only increase total hemoglobin mass in athletes with an initial total hemoglobin mass value.[11] Saunders et al quantified physiological and performance effects of hypoxic exposure, a training camp, the placebo effect, and a combination of these factors. Overall, 3-wk LHTL simulated altitude training for $14 \mathrm{~h} / \mathrm{d}$ increased Hbmass and $\mathrm{VO}_{2}$ peak, but the improvement in treadmill performance was not greater than 
the training camp effect. [12] Nummela \& Rusko investigated the benefits of living high and training low on anaerobic performance at sea level, eight 400-m runners lived for 10 days in normobaric hypoxia in an altitude house and trained outdoors in ambient normoxia at sea level. demonstrate improved 400-m performance after 10 days of living in normobaric hypoxia and training at sea level. Furthermore, their study provided evidence that changes in the acid-base balance and lactate metabolism might be responsible for the improvement in sprint performance. [13]

In the present study, the authors adopted the $75 \% \mathrm{VO}_{2 \max }$ exercise intensity to exercise for $45 \mathrm{~min}$ and then to increase the exercise intensity to $90 \% \mathrm{VO}_{2 \max }$ and up to exhausted exercise, by means of the comparison of the oxygen uptake, heart rate, power output, and oxhemoglobin saturation by pulse oximetry, blood lactic acid etc. in plain and plateau and make an attempt to give some suggestion for coaches and athletes on exercise intensity of altitude training.

\section{Method}

\section{Participant}

Eight middle and long distance runners in collegiate team affiliated Chengdu Sport University; the basic demographic information was listed in table 1.

Table 1. Demographic information in the study

\begin{tabular}{ccccccc}
\hline Gender & $\mathrm{N}$ & Age $(\mathrm{yrs})$ & Height $(\mathrm{cm})$ & Weight $(\mathrm{kg})$ & Training Years (yrs) & Living altitude $(\mathrm{m})$ \\
\hline Male & 5 & $23.5 \pm 1.2$ & $183.2 \pm 8.6$ & $76.2 \pm 4.3$ & $6.2 \pm 1.2$ & 503 \\
Female & 3 & $23.1 \pm 2.3$ & $168.5 \pm 3.5$ & $62.5 \pm 8.2$ & $8.5 \pm 3.2$ & 503 \\
\hline
\end{tabular}

\section{Experimental Design}

1) maximal oxygen uptake test: the test have been performed on June 4 to 7,2016 in Key laboratory of Chengdu Sport University (503m above sea level). The individual $\mathrm{VO}_{2 \max }$ was obtained using progressive increment of load $(15 \mathrm{~W} / \mathrm{min}$.) to the exhausted level. Within two weeks after the $\mathrm{VO}_{2 \max }$, the endurance tests have been carried out in Chengdu and Kangding.

2) Physiological and biochemical index test: during endurance test the oxygen uptake, heart rate, power output, and oxhemoglobin saturation by pulse oximetry, blood lactic acid were obtained.

\section{Data Analysis}

All collected data were analyzed using IBM SPSS Statistics 22.0, the description statistics, one-way ANOVOA, LSD multiple comparison were used in the analysis, and significant level was set at 0.05 .

\section{Results}

\section{$\mathrm{SPO}_{2}$ Differences in Plain and Plateau}

As listed in table 2, the results indicated that (1) the $\mathrm{SPO}_{2}$ value at all points were significant lower in Kangding plateau than that in Chengdu plain during the $75 \%$ $\mathrm{VO}_{2 \max }$ for $45 \mathrm{~min}$ and the following $90 \% \mathrm{VO}_{2 \max }$ to exhausted endurance test, (2) the $\mathrm{SPO}_{2}$ value after exhausted endurance test was significant lower than that at $5 \mathrm{~min}$. In Chengdu, however, the result was opposite in Kangding, (3) in general, the $\mathrm{SPO}_{2}$ values in Chengdu were significant lower than that in Kangding. 
Table 2. Change characteristic of $\mathrm{SPO}_{2}$ of endurance test between Chengdu and Kangding

\begin{tabular}{cccccc}
\hline & $5 \min (\mathrm{a})$ & $25 \min (\mathrm{c})$ & $45 \min (\mathrm{e})$ & Exhausted(f) & LSD multiple \\
\hline Chengdu & $97.06 \pm 1.77$ & $95.58 \pm 3.25$ & $95.88 \pm 4.14$ & $93.14 \pm 3.25$ & Paf* Pcf* Pef* Pae* \\
Kangding & $86.21 \pm 5.62$ & $87.98 \pm 6.14$ & $86.74 \pm 6.15$ & $89.14 \pm 4.69$ & Paf* Pcf* Pef* Pce* \\
T Test & $\mathrm{P}<0.05$ & $\mathrm{P}<0.05$ & $\mathrm{P}<0.05$ & $\mathrm{P}<0.05$ & \\
\hline
\end{tabular}

Note: ${ }^{*} \mathrm{p}<0.05$

\section{Heart Rate Differences in Plain and Plateau}

As listed in table 3, the result indicated that (1) at 5min., 25min.,45min., and exhausted time, there were insignificant differences in heart rate in endurance test,(2) both in Chengdu and Kangding, there were significant higher at 25min.,45min., and exhausted time than that at $5 \mathrm{~min}$.

Table 3. Change characteristic of heart rate of endurance test between Chengdu and Kangding

\begin{tabular}{cccccc}
\hline & $5 \min (\mathrm{a})$ & $25 \min (\mathrm{c})$ & $45 \min (\mathrm{e})$ & Exhausted(f) & LSD multiple \\
\hline Chengdu & $144.34 \pm 12.2$ & $162.49 \pm 15.5$ & $165.29 \pm 12.3$ & $185.58 \pm 12.7$ & Pac* Pae* Paf* Pcf* \\
Kangding & $149.21 \pm 14.1$ & $168.25 \pm 9.68$ & $168.41 \pm 10.3$ & $187.23 \pm 11.5$ & Pac* Pae* Paf* Pcf* $^{*}$ Paf \\
T test & $\mathrm{P}>0.05$ & $\mathrm{P}>0.05$ & $\mathrm{P}>0.05$ & $\mathrm{P}>0.05$ & \\
\hline
\end{tabular}

Note: ${ }^{*} \mathrm{p}<0.05$

\section{Power Output Differences in Plain and Plateau}

As listed in table 4 , the result indicated that (1) the power output at $25 \mathrm{~min}$. and $45 \mathrm{~min}$. were significant lower than that at 5min. both in Chengdu and in Kangding, and (2) the power output at any point in Kangding were lower than that in Chengdu.

Table 4. Change characteristic of power output of endurance test between Chengdu and Kangding

\begin{tabular}{|c|c|c|c|c|c|}
\hline & $5 \min (a)$ & $25 \min (c)$ & $45 \min (e)$ & Exhausted(f) & LSD multiple \\
\hline Chengdu & $122.15 \pm 15.7$ & $113.85 \pm 24.0$ & $111.69 \pm 23.7$ & $170.29 \pm 28.2$ & $\mathrm{Pac}^{*} \mathrm{Pae}^{*} \mathrm{Paf} *$ \\
\hline Kangding & $113.62 \pm 26.3$ & $106.49 \pm 19.1$ & $101.78 \pm 24.1$ & $152.69 \pm 26.4$ & $\mathrm{Pac}^{*} \mathrm{Pae}^{*} \mathrm{Paf}^{*}$ \\
\hline $\mathrm{T}$ test & $\mathrm{P}<0.05$ & $\mathrm{P}<0.05$ & $\mathrm{P}<0.05$ & $\mathrm{P}<0.05$ & \\
\hline
\end{tabular}

Note: ${ }^{*} \mathrm{p}<0.05$

\section{Blood lactic Acid Differences in Plain and Plateau}

As listed in table 5, the result indicated that there was no significant difference in blood lactic acid before endurance test both in Chengdu and in Kangding, the improvement of blood lactic acid was significant difference between pre- and post endurance test, however, there was no significant difference between Chengdu and Kanding post endurance test.

Table 5. Change characteristic of Blood lactic acid of endurance test between Chengdu and Kangding

\begin{tabular}{ccc}
\hline & PRE-BL & Post-BL \\
\hline Chengdu & $0.78 \pm 0.56$ & $5.21 \pm 1.66$ \\
Kangding & $1.02 \pm 0.39$ & $5.74 \pm 2.09$ \\
T Test & $\mathrm{P}>0.05$ & $\mathrm{P}>0.05$ \\
\hline
\end{tabular}




\section{Conclusions}

(1) The altitude training can reduce the power output and SPO2, the power output and heart rate were significant affected under long term altitude training.

(2) Heart rate and blood lactic acid seem have not been affected by training environmental factors.

(3) The findings of this study revealed that taking the heart rate as monitor criteria in moderate altitude training was an appropriate way, and the power output criteria may under- or over-estimate the exercise intensity in altitude training.

\section{Acknowledgment}

This research was financially supported by the Sichuan Science and Technology Support Project (NO. D60030105).

\section{References}

[1] Diebel, Sebastian R.; Newhouse, Ian; Thompson, David S.; Johson, Vineet B. K., The Effects of a 10-day Altitude Training Camp at 1828 Meters on Varsity Cross-Country Runners.International Journal of Exercise Science 2017, Vol. 10 Issue 1, p97

[2] Woods, Amy L.; Sharma, Avish P.; Garvican-Lewis, Laura A.; Saunders, Philo U.; Rice, Anthony J.; Thompson, Kevin G., Four Weeks of Classical Altitude Training Increases Resting Metabolic Rate in Highly Trained Middle-Distance Runners. International Journal of Sport Nutrition \& Exercise Metabolism Feb2017, Vol. 27 Issue $1, \mathrm{p} 83$

[3] Stickford, Abigail S. L.; Wilhite, Daniel P.; Chapman, Robert F.,No Change in Running Mechanics With Live High--Train Low Altitude Training in Elite Distance Runners.International Journal of Sports Physiology \& Performance Jan2017, Vol. 12 Issue $1, \mathrm{p} 133$

[4] Warren, Brian G.; Spaniol, Frank J.; Bonnette, Randy A., The Effects of an Elevation Training Mask on VO2max of Male Reserve Officers Training Corps Cadets.International Journal of Exercise Science 2017, Vol. 10 Issue 1, p37

[5] Bor-Kucukatay, Melek; Colak, Ridvan; Erken, Gülten; Kilic-Toprak, Emine; Kucukatay, Vural. Altitude training induced alterations in erythrocyte rheological properties: A controlled comparison study in rats. Clinical Hemorheology \& Microcirculation. 2014, Vol. 58 Issue 4, p479-488.

[6] Carr, Amelia J.; Saunders, Philo U.; Vallance, Brent S.; Garvican-Lewis, Laura A.; Gore, Christopher J., Increased Hypoxic Dose after Training at Low Altitude with 9h per Night at 3000m Normobaric Hypoxia. Journal of Sports Science \& Medicine Dec2015, Vol. 14 Issue 4, p776

[7] Siewierski, M.; Słomiński, P.; Białecki, R.; Adamczyk, J., Athletic Performance of Swimmers after Altitude Training (2,300 m Above Sea Level) in View of Their Blood Morphology Changes. Biology of Sport 2012, Vol. 29 Issue 2, p115

[8] Suchý, Jiří; Opočenský, Jakub, Usefulness of training camps at high altitude for well-trained adolescents. Acta Gymnica 2015, Vol. 45 Issue 1, p13 
[9] McLean, Blake D.; Buttifant, David; Gore, Christopher J.; White, Kevin; Liess, Carsten; Kemp, Justin, Physiological and Performance Responses to a Preseason Altitude-Training Camp in Elite Team-Sport Athletes. International Journal of Sports Physiology \& Performance Jul2013, Vol. 8 Issue 4, p391

[10] Garvican, L.; Martin, D.; Quod, M.; Stephens, B.; Sassi, A.; Gore, C.,Time course of the hemoglobin mass response to natural altitude training in elite endurance cyclists.Scandinavian Journal of Medicine \& Science in Sports Feb2012, Vol. 22 Issue $1, \mathrm{p} 95$

[11]Robach, P.; Lundby, C., Is live high-train low altitude training relevant for elite athletes with already high total hemoglobin mass? Scandinavian Journal of Medicine \& Science in Sports Jun2012, Vol. 22 Issue 3, p303

[12] Saunders, Philo U.; Ahlgrim, Christoph; Vallance, Brent; Green, Daniel J.; Robertson, Eileen Y.; Clark, Sally A.; Schumacher, Yorck O.; Gore, Christopher J., An Attempt to Quantify the Placebo Effect From a Three-Week Simulated Altitude Training Camp in Elite Race Walkers. International Journal of Sports Physiology \& Performance Dec 2010, Vol. 5 Issue 4, p521

[13] Nummela, Ari; Rusko, Heikki, Acclimatization to altitude and normoxic training improve 400-m running performance at sea level. Journal of Sports Sciences Jun 2000, Vol. 18 Issue 6, p411 